\title{
An Approximate E-Bayesian Estimation of Step-stress Accelerated Life Testing with Exponential Distribution
}

\author{
Liping $\mathrm{Hu}^{1}$ and Xianbin $\mathrm{Wu}^{2}, *$ \\ ${ }^{I}$ College of Information and Management, Henan Agriculture University, Zhengzhou, 450002, China; ${ }^{2} J u n i o r ~ C o l l e g e$, \\ Zhejiang Wanli University, Ningbo, Zhejiang, 315100, China
}

\begin{abstract}
The data analysis problem of step-stress accelerated life testing with exponential distribution is discussed. At the step-stress accelerated life testing conditions, an approximate E-Bayesian parameter estimation of step-stress accelerated life testing with exponential distribution is given by considering the prior distributions of the hyperparameters and using Gibbs sampling method. Finally, a simulation example is given, the results show that the Gibbs sampling method is simple and the convergence is better. E-Bayesian parameter estimation is more effective than the maximum likelihood estimation.
\end{abstract}

Keywords: E-Bayesian estimation, step-stress life testing, exponential distribution, Gibbs sampling.

\section{INTRODUCTION}

With the development of technology and the improvement of the products quality, high reliability and long-life products are available everywhere. However, at normal working conditions, the implementation of life testing can not meet the requirements of reliability evaluation. Accelerated life testing is a life testing method that is used to shorten the life testing cycle by increasing the stresses. Accelerated life testing method can be used to assess the reliability of the products in a relatively short period of time and to identify the reasons for product failure. Accelerated life testing data analysis and parameter estimations are theoretical and practical application value.

Step-stress accelerated life testing (briefly step-stress life testing) is an important life testing of accelerated life testing. In recent years, using the given statistical model of stepstress life testing with exponential distribution, the paper [1] gave the statistical analysis method for type IIcensoring life testing samples; the paper [2] gave the necessary and sufficient condition for the existence and uniqueness of the MLE of the step-stress life testing in typeI and II censoring cases and got the approximate confidence interval of the mean life at the normal stress level on that basis; in type II censoring case, the paper [3] got the Bayesian parameter estimation with constraints of step-stress life testing under the exponential distribution; the paper [4] gave an approximate Bayesian parameter estimation for step-stress life testing under the exponential distribution; the paper [5] gave a hierarchical Bayesian parameter estimation for step-stress life testing under the exponential distribution.

Although in typeII censoring case, the Bayesian parameter estimation for step-stress life testing with exponential distribution was given in the papers [3-5], on the one hand there is no a good consideration on the parameters in the prior distributions; on the other hand the calculation of the posterior marginal distribution function involves complex integral calculations. Based on the above considerations, the E-Bayesian method is given for parameter estimation of step-stress life testing with exponential distribution in this paper. Prior distributions of the hyperparameters in the prior distribution are also given, thus the joint posterior density function is got. For the calculation of the parameter estimation in the joint posterior density function, Gibbs sampling is used for the iteration of parameters to be estimated. Finally, a simulation example is given to analog comparator the EBayesian estimation and the maximum likelihood estimation, the results show that the E-Bayesian estimation is more effective than the maximum likelihood estimation.

This paper is organized as follows: In Section 2, the basic assumptions for step-stress life testing are stated. E-Bayesian estimations of step-stress life testing parameters are stated in Section 3. The estimation of the reliability indexes with exponential distribution in Section 4. An example is given to illustrate the proposed procedure in Section 5. The conclusion of this study is given in Section 6 .

\section{THE BASIC ASSUMPTIONS OF STEP-STRESS LIFE TESTING}

Determine the normal stress level $S_{0}$ and the accelerated stress levels $S_{1}, S_{2}, \ldots, S_{k}$, the stress levels meet $S_{0}<S_{1}<S_{2}<$ $\ldots<S_{k}, n$ samples are taken from a number of products for step-stress life testing.

At the stress level $S_{i}$, the working time of the failure products are $0 \leq t_{i 1} \leq \mathrm{t}_{i 2} \leq \ldots t_{i_{i}} \leq \tau_{i}$. In the case of typeI censoring, $\tau_{i}$ is the pre-given time for stopping the tests at the stress level $S_{i}, r_{i}$ is the number of failure products before the time $\tau_{i}$ at the stress level $S_{i}$; in the case of typeII censoring, $r_{i}$ is the pre-given number of samples for stopping the tests. 
Assumption 1. At the normal stress level $S_{0}$ and the accelerated stress levels $S_{1}<S_{2}<\ldots<S_{k}$, the life distribution of a test unit all obey the exponential distribution, its cumulative distribution function is:

$$
F_{i}\left(t \mid S_{i}\right)=1-\exp \left[-\lambda_{i}\left(S_{i}\right) t\right], t>0
$$

where $\lambda_{i}\left(S_{i}\right)>0$ is the failure rate of the products at the stress level $S_{i}$, its mean life is

$$
\theta_{\mathrm{i}}\left(\mathrm{S}_{\mathrm{i}}\right)=1 / \lambda_{\mathrm{i}}\left(\mathrm{S}_{\mathrm{i}}\right)
$$

Assumption 2. The mean life of the products: $\theta_{i}$ is the accelerated life function of the stress:

$\ln \theta_{i}=\mu+\beta \varphi\left(S_{i}\right)$

where $\mu, \beta$ is parameters to be estimated; $\varphi\left(S_{i}\right)$ is the known function of the stress level $S_{i}$.

Assumption 3. Residual life of the products depends only on the already cumulative failure part and the stress level at that time, but has nothing to do with the cumulative [6].

\section{E-BAYESIAN ESTIMATION OF STEP-STRESS LIFE TESTING PARAMETERS}

\subsection{The Likelihood Function of Step-stress Life Testing Parameters}

For step-stress life testing data with exponential distribution, the failure data $t_{11}, t_{12}, \ldots, t_{1} r_{1}$ are the life data of the samples at the stress level $S_{1}$; but the failure data is not the real life of the samples at the stress level $S_{i}$ when $i>1$. Therefore, the failure data need to be converted into the real life data.

According to Assumption 3, the cumulative failure probability at the stress level $S_{i}$ when the samples' working time is $t_{i}$ equivalent to the cumulative failure probability at the stress level $S_{j}$ when the samples' working time is $t_{i j}$. That is to say:

$$
F_{S_{i}}\left(t_{i}\right)=F_{S_{j}}\left(t_{i j}\right) i, j=1,2, \ldots, k .
$$

Then according to assumption one, we can get:

$$
1-\exp \left[-\lambda_{i}\left(S_{i}\right) t_{i}\right]=1-\exp \left[-\lambda_{j}\left(S_{j}\right) t_{i j}\right]
$$

So,

$$
t_{i j}=\frac{\lambda_{i t}}{\lambda_{j}}, i, j=1,2, \ldots, k
$$

Let $R_{i}=\sum_{j=1}^{i} r_{j}, i=1,2, \ldots, k$, so the total testing time at the stress level $S_{i}$ is:

$$
\begin{aligned}
& T_{i}=\sum_{j=1}^{r_{i}} t_{i j}+\left(n-R_{i}\right) \tau_{i},(\text { typeI Censoring case }) \\
& T_{i}=\sum_{j=1}^{r_{i}} t_{i j}+\left(n-R_{i}\right) t_{i_{r_{i}}},(\text { typeII Censoring case })
\end{aligned}
$$

Thus according to relevant theorems, the likelihood function is got:

$$
L\left(D \mid \lambda_{1}, \lambda_{2}, \ldots, \lambda_{k}\right) \propto \prod_{i=1}^{k} \lambda_{i} r_{i} \exp \left(-\sum_{i=1}^{k} \lambda_{i} T_{i}\right)
$$

where $\quad D=\left\{t_{11}, \ldots, t_{1_{r_{1}}}, \quad t_{21}, \ldots, t_{2_{r_{2}}}, \ldots, t_{k 1}, \ldots, t_{k_{r_{k}}}\right\}$, $\lambda_{1} \leq \lambda_{2} \leq \ldots \leq \lambda_{k}$.

According to Assumption 2, for $\lambda_{i}=1 / \theta_{i}$, we can see:

$$
\lambda_{i}=\lambda_{0} \exp \left\{b\left(\varphi\left(S_{0}\right)-\varphi\left(S_{i}\right)\right)\right\}=\lambda_{0} \alpha \psi_{i}
$$

where $\lambda_{0}$ is the failure rate of the products at the normal stress level; $\alpha=\exp \left\{b\left[\varphi\left(S_{0}\right)-\varphi\left(S_{1}\right)\right]\right\}=\lambda_{1} / \lambda_{0} \quad$ is the accelerating factor between the stress level $S_{1}$ and $S_{0}$, $\alpha>_{1} ; \psi_{i}=\frac{\varphi\left(S_{0}\right)-\varphi\left(S_{i}\right)}{\varphi\left(S_{0}\right)-\varphi\left(S_{1}\right)}, i=1,2, \ldots k$.

$$
\text { Let } T_{1}=\sum_{i=1}^{k} \psi_{i} r_{i}, T_{2}=\sum_{i=1}^{k} \alpha \psi_{i} T_{i}, r=\sum_{i=1}^{k} r_{i}
$$

So the likelihood function (3) can be converted to:

$$
L\left(\lambda_{0}, \alpha\right)=\lambda_{0}{ }^{r} \alpha T_{1} \exp \left(-\lambda_{0} T_{2}\right)
$$

Now, there are only two parameters in the likelihood function (3.2). In the actual production, people are most concerned about the failure rate at the normal stress level $\lambda_{0}$ and the acceleration factor $\alpha$.

\subsection{Definition of E-Bayesian Estimation}

Definition 1 [7]. With $\hat{\lambda}_{i}(a, b)$ being continuous,

$$
\hat{\lambda}_{\mathrm{iE}}=\iint_{D} \hat{\lambda}_{i}(a, b) \pi(a, b) d a d b=E\left[\hat{\lambda}_{i}(a, b)\right]
$$

is called the expected Bayesian estimation of $\lambda_{i}$ (briefly E-Bayesian estimation), where $\hat{\lambda}_{i}(a, b)$ is Bayesian estimation of $\lambda_{i}$ with hyperparameters $a$ and $b, D$ is the domain of $(a, b)$, and $\pi(a, b)$ is the density function of $a$ and $b$ over $D$.

By Definition 1, the E-Bayesian estimation of $\lambda_{i}$ is the expectation of the Bayesian estimation of $\lambda_{i}$ for the hyperparameters $a$ and $b$. The E-Bayesian estimation of $\lambda_{i}$ is not Bayesian estimation or hierarchical Bayesian estimation [8], it can be seen as a kind of modified Hierarchical Bayesian estimation. The E-Bayesian estimation method have wide scope potential applications in many fields $[9,10]$.

\subsection{The Prior Distribution of the Parameters $\lambda_{0}, \alpha$ and the Joint Posterior Density Function}

Based on the engineering experience, the range of the acceleration factor: $\alpha$ is $1 \leq k_{1}<\alpha<k_{2}$, for this, take the prior density function of $\alpha$ as: 


$$
\pi(\alpha)=\alpha^{-1}, 1 \leq k_{1}<\alpha<k_{2}
$$

If the prior distribution of $\lambda_{\mathrm{o}}$ be its conjugated distribution-Beta $(a, b)$ with density function as follows:

$$
\pi\left(\lambda_{0} \mid a, b\right)=\frac{\lambda_{0}{ }^{a-1}\left(1-\lambda_{0}\right)^{b-1}}{B(a, b)}
$$

where $0<\lambda_{0}<\infty, a>0, b>0, B(a, b)=\int_{0}^{1} t^{a-1}(1-t)^{b-1} \mathrm{~d} t$ is the Beta function, $a>0, b>0$, both $a$ and $b$ are hyperparameters

In the case of modern high-reliability products, the possibility of the failure rate $\lambda_{\mathrm{o}}$ being larger is smaller than the possibility of the failure rate $\lambda_{\mathrm{o}}$ being smaller. For this, according to the paper [8], $a$ and $b$ should be chosen so that $\pi\left(\lambda_{0} \mid a, b\right)$ is a decreasing function of $\lambda_{0}$. When $0<a<1,1<b$, $\pi\left(\lambda_{0} \mid a, b\right)$ is a decreasing function of $\lambda_{0}$. To determine the specific value of $a, b$ is very difficult, for the two hyperparameters are unobservable and the information obtained in practical application is insufficient to determine the value of $a, b$. Therefore, a uniform distribution can be respectively defined over the range of $a$ and $b$ as the prior distributions of hyperparameters $a$ and $b$. For this, take the prior distributions of hyperparameters $a, b$ as follows:

$$
\pi_{1}(a)=U(0,1), \pi_{2}(b)=U(1, c)
$$

where $c$ is a constant.

Considering that in the case of $a<1$, the bigger $b$ is, the thinner is the tail of the Beta density function. But in view of the robustness of the Bayesian estimation, the thinner tailed prior distribution often leads to the worse robustness of the Bayesian estimate. Accordingly, $b$ should not be too big, it is better to be chosen below some given upper bound $c(c>1$ is a constant to be determined)

When the parameters $a, b$ is of independence, according to the Definition 1 , we can get the prior density function of $\lambda_{0}$ :

$$
\pi\left(\lambda_{0}\right)=\frac{1}{c-1} \int_{1}^{c} \int_{0}^{1} \frac{\lambda_{0}{ }^{a-1}\left(1-\lambda_{0}\right)^{b-1}}{B(a, b)} \mathrm{d} a \mathrm{~d} b
$$

So by the prior density function of $\alpha$ and $\lambda_{0}$ : (3.4) and (3.5) , as well as the likelihood function (3.2), according to Bayesian theorem, we can get its joint posterior density function, then according to Definition 1, we can get the EBayesian joint posterior density function of $\left(\lambda_{0}, \alpha, a, b\right)$ :

$$
\begin{aligned}
& \pi\left(\lambda_{0}, \alpha, a, b \mid T\right) \propto \frac{1}{B(a, b)} \lambda_{0}^{r+a-1} \\
& \left(1-\lambda_{0}\right)^{b-1} \alpha^{T_{1}-1} \exp \left(-\lambda_{0} T_{2}\right)
\end{aligned}
$$

where $T=\left(n, r_{i}, \tau_{i}, t_{i j}, j=1,2, \ldots, r_{i}, i=1,2, \ldots, k\right)$

\subsection{The E-Bayesian Estimation of the Parameters $\lambda_{0}$ and $\alpha$}

The problem of using Bayesian method for statistical inference is the calculation of the posterior marginal distribu- tion function. In many cases, it is difficult or even impossible to obtain the analytic expression of the posterior marginal distribution function, sometimes we can get the analytic expression but the results are rather complicated that is not easy for application and promotion. Therefore, this paper uses Gibbs sampling approach for the iteration of parameters to be estimated, the mean of the parameters to be estimated is obtained. The biggest advantage of the approach is implementing simple and with the convergence of iteration.

\subsubsection{Gibbs Sampling}

MCMC (Markov Chain Monte Carlo) method is through the establishment of the Markov chain with a stable distribution to obtain the samples of $p\left(\theta \mid x_{n}\right)$, then make statistical inference for the samples obtained. One of the most simple and extensive application of MCMC methods is Gibbs sampling method. It was proposed by $S$. Geman and D. Geman in 1984 [11].

Take random variables as $\theta_{1}, \theta_{2}, \ldots, \theta_{k}$, assume its full conditional distribution $\mathrm{p}\left(\theta_{s} \mid \theta_{r}\right)(r \neq s), s=1,2, \ldots, k$ is available sampling, that is to say, when a set of values for random variable $\theta_{r}(r \neq s)$ are given, we can generate a random sample of $\theta_{s}$. The paper [12] proved that at appropriate conditions, the joint distribution function $\mathrm{p}\left(\theta_{1}, \theta_{2}, \ldots, \theta_{k}\right)$ was only decided by the full conditional distribution, so all of the marginal distribution function $p\left(\theta_{s}\right), s=1,2, \ldots, k$ are only determined by the full conditional distribution.

Gibbs sampling process is as follows: the starting point is given $\theta^{(0)}=\left(\theta_{1}{ }^{(0)}, \theta_{2}{ }^{(0)} \ldots, \theta_{k}{ }^{(0)}\right)$,

(1) Sampling $\theta_{1}{ }^{(1)}$ from the full conditional distribution $\mathrm{p}\left(\theta_{1} \mid x_{n}, \theta_{2}{ }^{(0)}, \ldots, \theta_{k}^{(0)}\right)$;

(2) Sampling $\theta_{2}^{(1)}$ from the full conditional distribution $p\left(\theta_{2} \mid x_{\mathrm{n}}, \theta_{1}{ }^{(1)}, \theta_{3}{ }^{(0)}, \ldots, \theta_{k}{ }^{(0)}\right)$;

$\ldots$;

(i) Sampling $\theta_{1}^{(1)}$ from the full conditional distribution $p\left(\theta_{\mathrm{i}} \mid x_{n},{\theta_{1}}^{(1)}, \theta_{2}^{(1)}, \ldots, \theta_{i-1}{ }^{(1)}, \theta_{i+1}{ }^{(0)}, \ldots, \theta_{k}^{(0)}\right)$;

...;

(k) Sampling $\theta_{k}^{(1)}$ from the full conditional distribution $p\left(\theta_{k} \mid x_{n}, \theta_{1}^{(1)}, \ldots, \theta_{k-1}{ }^{(1)}\right)$.

Repeat the above (1) to $(k)$ steps, after $t$-steps iterations; we can get a Markov chain:

$$
\begin{aligned}
& \theta^{(1)}=\left(\theta_{1}{ }^{(1)}, \theta_{2}{ }^{(1)} \ldots, \theta_{k}{ }^{(1)}\right) ; \\
& \theta^{(2)}=\left(\theta_{1}^{(2)}, \theta_{2}^{(2)} \ldots, \theta_{k}^{(2)}\right) \\
& \ldots ; \\
& \theta^{(t)}=\left(\theta_{1}^{(t)}, \theta_{2}^{(t)} \ldots, \theta_{k}^{(t)}\right)
\end{aligned}
$$

The sample which make Markov chain reach equilibrium can be as a sample of $p\left(\theta \mid x_{n}\right)$.

The fact can be proved that at appropriate conditions, when Iteration times $t \rightarrow \infty$, then

$$
p\left(\theta_{1}{ }^{(t)}, \theta_{2}^{(t)} \ldots, \theta_{k}^{(t)}\right) \rightarrow p\left(\theta_{1}, \theta_{2}, \ldots, \theta_{k}\right)
$$


Hence, an estimation of the marginal distribution we need can be got [13]:

$$
\hat{f}\left(\theta_{s}\right)=f\left(\theta_{s} \mid \theta_{r}{ }^{(t)}, r \neq s\right) .
$$
by $t$.

The convergence of the iteration sampling is determined

\subsubsection{The Gibbs Sampling Process of the Parameters $\lambda_{0}$, $\alpha$, a and $\mathrm{b}$}

By the E-Bayesian joint posterior density function (3.6), we can see that the full conditional posterior probability density function of $\lambda_{0}$ is:

$$
\pi\left(\lambda_{0} \mid \alpha, a, b, T\right) \propto \lambda_{0}^{r+a-1}\left(1-\lambda_{0}\right)^{b-1} \exp \left(-\lambda_{0} T_{2}\right)
$$

The full conditional posterior probability density function of $\alpha$ is:

$$
\pi\left(\alpha \mid \lambda_{0}, a, b, T\right) \propto \alpha^{T_{1}-1} \exp \left(-\lambda_{0} T_{2}\right) .
$$
of $a$ is:

The full conditional posterior probability density function

$$
\pi\left(a \mid \alpha, \lambda_{0}, b, T\right) \propto \lambda_{0}{ }^{a} / B(a, b) .
$$
of $b$ is:

The full conditional posterior probability density function

$$
\pi\left(b \mid \alpha, \lambda_{0}, a, T\right) \propto\left(1-\lambda_{0}\right)^{b} / B(a, b) .
$$

The random number for the full conditional posterior distribution of $\lambda_{0}, \alpha, a$ and $b$ can be produced by selected sampling method.

According to the steps of Gibbs sampling method, the starting point is given as $\left(\lambda_{0}{ }^{(0)}, \alpha^{(0)}, a^{(0)}, b^{(0)}\right)$, then the $t$ time iteration is divided into the following four steps:

(1) Sampling $\lambda_{0}{ }^{(t)}$ from the full conditional posterior distribution $\pi\left(\lambda \mid T, \alpha^{(t-1)}, a^{(t-1)}, b^{(t-1)}\right)$.

(2) Sampling $\alpha^{(t)}$ from the full conditional posterior distribution $\pi\left(\alpha \mid T, \lambda_{0}{ }^{(t)}, a^{(t-1)}, b^{(t-1)}\right)$.

(3) Sampling $a^{(t)}$ from the full conditional posterior distribution $\pi\left(a \mid T, \lambda_{0}{ }^{(t),} \alpha^{(t),} b^{(t-1)}\right)$.

(4) Sampling $b^{(t)}$ from the full conditional posterior distribution $\pi\left(b \mid T, \lambda_{0}{ }^{(t)}, \alpha^{(t),} a^{(t)}\right)$.

Then $\left(\lambda_{0}{ }^{(t)}, \alpha^{(t)}, a^{(t)}, b^{(t)}, t=1,2, \ldots n, n+1, \ldots, N\right)$ is a Gibbs iteration sample of parameters $\left(\lambda_{0}, \alpha, a, b\right)$, where $n$ is the discarded sample size before Gibbs iterations have got a steady state, $N>_{n}$ is the overall sample size.

We only care about the failure rate at the normal stress level: $\lambda_{0}$ and the acceleration factor $\alpha$, so the E-Bayesian parameter estimation of $\lambda_{0}$ and $\alpha$ are respectively:

$$
\hat{\lambda}_{0}=\frac{1}{N-n} \sum_{t=n+1}^{N} \lambda_{0}{ }^{(t)}, \hat{\alpha}=\frac{1}{N-n} \sum_{t=n+1}^{N} \alpha^{(t)}
$$

\section{THE ESTIMATION OF THE RELIABILITY IN- DEXES WITH EXPONENTIAL DISTRIBUTION}

Our ultimate goal is to get the estimation of the product mean life at the normal stress level: $\theta_{0}$, then according to the assumption two and the accelerated life equation (2.1), the parameters $\mu$ and $\beta$ in the accelerated life equation are estimated. Thereby, the accelerated life model is established.

According to the Assumption 2, the mean life is $\theta_{i}\left(S_{i}\right)=1 / \lambda_{i}\left(S_{i}\right)$. So the first reliability index which is the mean life is got:

$$
\hat{\theta}_{0}=1 / \hat{\lambda}_{0}
$$

Using the E-Bayesian parameter estimation of the failure rate $\hat{\lambda}_{0}$, the second reliability index which is reliability is obtained:

$$
R(t)=\exp (-\lambda t)=\exp \left(-\hat{\lambda}_{0} t\right)
$$

Then according to the accelerated life equation (1), we have $\ln \hat{\boldsymbol{\theta}}_{0}=\mu+\beta \varphi\left(S_{0}\right)$, using the least square method to get a distribution curve with various points $(\mu, \beta)$, the approximation of the parameters $\mu$ and $\beta$ can be estimated $: \mu=\hat{\mu}$, $\beta=\hat{\beta}$. Thereby, we can establish the accelerated life model:

$$
\ln \theta_{i}=\hat{\mu}+\hat{\beta} \varphi\left(\mathrm{S}_{i}\right) .
$$

\section{A SIMULATION EXAMPLE}

Now there are a number of electronic products, their life obeys the exponential distribution, 40 samples are taken from the products for the four-steps step-stress accelerated life testing. The normal stress level is $S_{0}=28 \mathrm{~V}$ (volt), take accelerated stress levels as $S_{1}=38 \mathrm{~V}, S_{2}=41 \mathrm{~V}, S_{3}=44 \mathrm{~V}$, $S_{4}=47 \mathrm{~V}$, the censoring time of each step is $\tau_{1}=1000 \mathrm{~h}$ (hour), $\tau_{2}=600 \mathrm{~h}, \tau_{3}=250 \mathrm{~h}, \tau_{3}=125 \mathrm{~h}$. Accelerated life equation $\ln \theta=68-16 \ln S$ is Pre-given, where the parameters $\mu=68, \beta=-$ 16. Using Monte-Carlo method to obtain a set of lifetime data:

First using the method in the paper [2] parameters $\mu, \beta$ in the accelerated life equation are estimated, the MLE of $\mu, \beta$ are respectively: $\hat{\mu}=61.2955, \hat{\beta}=-14.2498$. Then from the accelerated life equation, we can get the estimation of the failure rate: $\hat{\lambda}_{0}=1.0033 \times 10^{-6}$ and the estimation of the acceleration factor: $\hat{\alpha}=77.6047$.

Next, E-Bayesian method is used to estimate the above parameters. Take the uniform distribution over $(100,150)$ as the prior distribution of $\alpha$, take Gibbs sampling iteration times $N=5500$, the initial value are given as $\lambda_{0}=0.1, \alpha=100$, $a=0.1, b=20$. The fact can be found that the parameters basically reach a steady state after the 500 iteration steps .Therefore, the E-Bayesian estimation of the parameters $\lambda_{0}$ and $\alpha$ are obtained from the mean of 5000 samples after $n=$ 500 steps, then we can get the estimation of the failure Rate: $\hat{\lambda}_{0}=4.3107 \times 10^{-7}$ and the estimation of the accelera- 
Table 1. Step-stress life testing data of the electronic products.

\begin{tabular}{|c|c|c|c|c|c|}
\hline$t_{11}$ & & & & & \\
\hline $921.2852 \mathrm{~h}$ & & & & $t_{25}$ & \\
\hline$t_{21}$ & $t_{22}$ & $t_{23}$ & $t_{24}$ & $t_{35}$ \\
\hline $50.4314 \mathrm{~h}$ & $104.2676 \mathrm{~h}$ & $245.5130 \mathrm{~h}$ & $450.5856 \mathrm{~h}$ & $t_{36}$ & $214.0247 \mathrm{~h}$ \\
\hline$t_{31}$ & $t_{32}$ & $t_{33}$ & $179.0157 \mathrm{~h}$ & \\
\hline $49.7372 \mathrm{~h}$ & $67.1302 \mathrm{~h}$ & $112.7626 \mathrm{~h}$ & $t_{44}$ & \\
\hline$t_{41}$ & $t_{42}$ & $t_{43}$ & $112.0713 \mathrm{~h}$ & $115.0968 \mathrm{~h}$ & \\
\hline $50.7118 \mathrm{~h}$ & $103.6320 \mathrm{~h}$ & & & \\
\hline
\end{tabular}

tion factor: $\hat{\alpha}=129.2715$, furthermore, we can get the estimation of parameters $\mu$ and $\beta$ are respectively $\hat{\mu}=67.7083$, $\hat{\beta}=-15.9208$.

The results show that E-Bayesian parameter estimations are very close to the true values; however the parameter estimations which are obtained by the MLE method have a larger difference with the true values.

\section{CONCLUSION}

Using the E-Bayesian method, the parameters estimation and the reliability indexes estimation for step-stress accelerated life testing with exponential distribution are given. The values of the hyperparameters in the prior distribution are avoided. Using Gibbs sampling for the calculation of the posterior marginal distributions and parameter estimation, the calculation with high-dimensional integrals is avoided. Finally, the fact can be seen that E-Bayesian parameter estimation is more effective and practical than the maximum likelihood estimation from the simulation example.

\section{CONFLICT OF INTEREST}

The authors confirm that this article content has no conflict of interest.

\section{ACKNOWLEDGEMENTS}

This work was financially supported by the Natural Science Foundation of Ningbo Grant (No. 2013A610276) and the Scientific Research Project from Education of Zhejiang Province (No. Y201431585) and the think-tank Entrepreneurship project of Ningbo(013B70066).

\section{REFERENCES}

[1] J. S. Liu, "Monte Carlo strategies in scientific computing," Springer-Verlag, New York, 2001.

[2] H. L. Fei and X. X. Zhang, "Maximum likelihood estimate of stepstress accelerated life testing under the exponential distribution," Chinese Mathematical Application, vol. 17, no. 3, pp. 398-404, 2004.

[3] C. X. Zhong and S. S. Mao, "Bayesian approach to an accelerated life test under the exponential distribution case," Chinese Applied Mathematics, vol. 8, no. 4, pp. 376-85, 1993.

[4] Z. H. Zhang, "Bayes analysis of step-stress accelerated life testing under exponential distributions," Chinese Applied Mathematics, vol. 12, no. 2, pp. 175-181, 1997.

[5] D. Wu and Y.C. Tang, "Bayesian estimation of step-stress accelerated life testing of exponential distribution under CE model," Chinese Application of Statistics and Management, vol. 27, no. 3, pp. 423-427, 2008 .

[6] W Nelson, "Accelerated life testing-step-stress models and data analysis," IEEE Trans Reliability, vol. 29, no. 2, pp. 103-108, 1980

[7] M. Han, "E-Bayesian estimation of failure probability and its application," Mathematical and Computer Modeling, vol. 45, pp. 1272-1279, 2007.

[8] M. Han, "The structure of hierarchical prior distribution and it's applications," Chinese Operations Research and Management Science, vol. 16, no. 3, pp. 31-40, 1997.

[9] G. L. Cai, L. L. Wu and X. F. Tang, "E-Bayes reliability analysis of double hyperparameters zero failure data," Journal of Jiangsu University (Natural Science Edition), vol. 31, no. 6, pp. 2010

[10] G. L. Cai and W. Q. Xu, "Application of E-Bayes method in stock forecast," Proceedings of The 4th International Conference on Information and Computing Science, vol. 1, pp. 504-506, 2011.

[11] Y. C. Tang and H. L. Fei, "Bayesian analysis for Weibull distribution accelerated life testing based on Gibbs sampling," Chinese Mathematical Statistics and Applied Probability, vol. 13, no. 1, pp. 81-88, 1998

[12] J. L. Besag, "Spatial interaction and the statistical analysis of lattice systems," Journal of the Royal Statistical Society. Series B (Methodological), vol. 36, no. 2, pp. 192-236, 1974.

[13] G. Stuart and G. Donald, "Stochastic Relaxation, Gibbs distributions and the Bayesian restoration of images," Journal of Applied Statistics, vol. 20, no. 6, pp. 25-62, 1993.

(C) $\mathrm{Hu}$ and $\mathrm{Wu}$; Licensee Bentham Open.

This is an open access article licensed under the terms of the Creative Commons Attribution Non-Commercial License (http://creativecommons.org/licenses/by-nc/3.0/) which permits unrestricted, non-commercial use, distribution and reproduction in any medium, provided the work is properly cited. 